\title{
Using rotation measure to search for magnetic fields around galaxies at $\mathrm{z} \sim 0.5$
}

\author{
Anna Williams ${ }^{1}$, Britt Lundgren ${ }^{2}$, Sui Ann Mao $^{3}$, Eric Wilcots ${ }^{1}$ and \\ Ellen Zweibel ${ }^{1}$ \\ ${ }^{1}$ Dept. of Astronomy, University of Wisconsin-Madison \\ Madison, Wisconsin, USA \\ email: williams@astro.wisc.edu, ewilcots@astro.wisc.edu, zweibel@astro.wisc.edu \\ ${ }^{2}$ Dept. of Physics, University of North Caroline-Asheville \\ Asheville, NC, USA \\ email: blundgre@unca.edu \\ ${ }^{3}$ Max-Planck-Instititut Für Radioastronomie \\ Bonn, Nordrhein-Westfalen, DE \\ email: mao@mpifr-bonn.mpg.de
}

\begin{abstract}
Magnetic fields are an important component in galaxies, and yet, we still do not know how these magnetic fields were originally seeded within galaxies, nor how they have grown to the strengths we observe today. One way we can unravel this complex problem is by measuring the growth of magnetic fields over cosmic time. We present the initial results of a rotation measure study to search for the presence of coherent magnetic fields around young disk-like galaxies at $\mathrm{z} \sim 0.5$. The S-band receiver at the VLA allows us to simultaneously observe Stokes I, Q, U, and $\mathrm{V}$ from 2-4 GHz. With these broadband polarization observations we apply multiple methods for determining the rotation measure of each source, improving the fidelity of our results. Beyond magnetogenesis, the results of this study also have implications for the life-cycle of baryons within galaxies and the composition of galactic haloes.
\end{abstract}

Keywords. extragalactic, magnetic fields, MgII absorber, redshift

\section{Introduction}

Magnetic fields permeate the universe. They are present on all scales, from planets up to galaxy clusters. Despite their ubiquity, there are still unanswered questions surrounding the origin and evolution of astrophysical magnetic fields. These include how and when did the first magnetic fields form in the universe? how do the strength and structure of magnetic fields evolve with redshift? and are there magnetic fields in the intergalactic medium? Theory and simulations continue to improve our knowledge of the dynamo processes key to the production of magnetic fields, and recent upgrades to available radio telescopes allow us to obtain simultaneous wide-band polarization coverage. These new instruments are ideal for observing magnetic fields via Faraday rotation.

Faraday rotation, or rotation measure (RM), is due to the birefringence property of magnetized plasma which causes the plane of polarization to rotate as a function of wavelength. This is described by $\Delta \theta=\lambda^{2} 0.812 \int_{\text {source }}^{\text {observer }} n_{e} B d l$. Here, $\Delta \theta$ is the change in angle of linear polarization in rad, $\lambda^{2}$ is the wavelength of observation squared in $\mathrm{m}^{2}$, $n_{e}$ is the electron density in $\mathrm{cm}^{-3}, B$ is the strength of the line of sight magnetic field in $\mu \mathrm{G}$, and $d l$ is the distance traveled by the emission in pc.

In principle, the magnetic field strength can be determined by measuring the change in polarization angle across different wavelengths, and if there is information about the electron density and path length. In practice, it is a bit more complicated since the 
Figure 1. Examples of sightlines to distant QSOs. The top sightline is an example of an ideal control QSO with no intervening extragalactic systems contributing to the observed RM ( $\left.\mathrm{RM}_{\text {Obs. }}\right)$. The only components along the line of sight are the Milky Way $\left(\mathrm{RM}_{M W}\right)$, intergalactic medium $\left(\mathrm{RM}_{I G M}\right)$, gas associated with the QSO $\left(\mathrm{RM}_{Q S O}\right)$, and the uncertainty $(\sigma)$. The bottom sightline is an example of an ideal target QSO. In this case the $\mathrm{RM}_{O b s}$. also contains the $\mathrm{RM}$ associated with the intervening galaxy $\left(\mathrm{RM}_{I N T}\right)$.
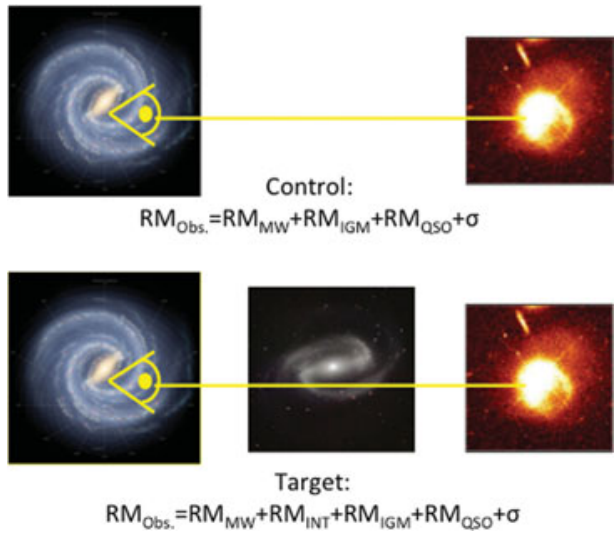

measured RM is the sum of all RM contributions along the line of sight. If one is looking for the change in RM as a function of redshift to measure the evolution of magnetic fields, this complexity is increases as is shown in Figure 1. To overcome the degeneracy involved in isolating the Faraday rotation associated with a particular component along the line of sight, large, well designed RM surveys are needed.

\section{Searching for magnetic fields near evolving galaxies at $z \sim 0.5$}

Bernet et al. (2008) found observational evidence of a redshift evolution in $|\mathrm{RM}|$ when they discovered that QSOs with strong MgII absorption have larger $|\mathrm{RM}|$. Mg II absorption probes a wide range of astrophysical phenomena including gas in the disks and halos of galaxies, high velocity clouds, dwarf galaxies, or even galactic outflows (e.g., Zibetti et al. (2007), Rubin et al. 2010). A synthesis of our current understanding of $\mathrm{Mg}$ II absorbers is that these systems provide an opportunity to understand the conditions in and around evolving galaxies. Farnes et al. (2014) came to a similar conclusion after compiling polarization data from all available radio surveys. While these results give tantalizing hints that MgII absorbers host coherent fields, their interpretation is hampered by the heterogenous nature of their samples, limited narrow-band data, and the general issue that we know little about how the background QSOs contribute to measurements of extragalactic RMs.

We are conducting a new and carefully defined survey of QSO RMs that probe the MgII absorbing material surrounding galaxies at $z \sim 0.5$. The primary goal of our survey is to constrain when coherent magnetic fields arose in the universe by determining if young, disk-like galaxies at $z \sim 0.5$ have associated fields. As was done in previous studies, we have selected two samples of QSOs: a 'control sample' with 112 QSOs that have no MgII absorption in their available optical spectra and a 'target sample' of 38 QSOs with a single MgII absorption system within a redshift range of $0.38<\mathrm{z}<0.65$. (see Figure 1 ). One way that our survey is an improvement over previous studies is that all of our MgII absorption systems have photometric detections in the Sloan Digital Sky Survey (SDSS) which allow us to analyze the effects of impact parameter. By utilizing the full wide-band coverage offered by the Karl G. Jansky Very Large Array (VLA) at S-band $(2-4 \mathrm{GHz})$, we are able to implement multiple advanced tools like RM synthesis Brentjens $\&$ de Brun (2005) and QU-fitting O'Sullivan et al. (2012) to determine more robust RMs than was previously possible. S-band also avoids longer wavelengths $(\leqslant 1.4 \mathrm{GHz})$ which are more susceptible to depolarization effects Schnitzeler et al. (2015). 


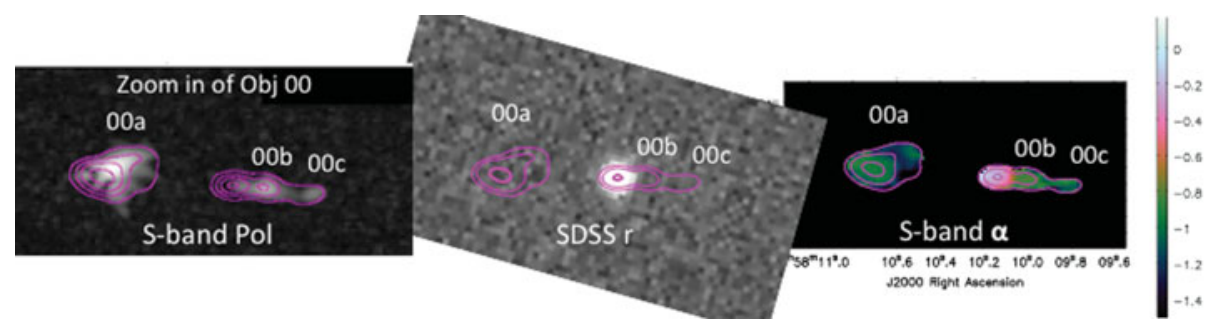

Figure 2. Three zoomed in images of a control source. In each zoom-in the contours trace the total intensity of the VLA observations integrated across the entire S-band (RMS $=20 \mu \mathrm{Jy}$ ), $2-4 \mathrm{GHz}$. The left image shows the polarization in greyscale. The middle image shows the SDSS r-band image in greyscale, and the right image shows the spectral index, $\alpha$ where $S_{\nu} \propto \nu^{\alpha}$, as described by the colorbar.

\section{Results}

Figure 2 shows an image of one of the control QSOs. The left greyscale image shows the polarized intensity at S-band, the middle greyscale image is the SDSS intensity map at $r$, and the right image and corresponding colorbar show the spectral index, $\alpha$, across the source. All three images show the S-band total intensity contours overplotted in magenta. The three brightest points of polarization have been labeled $00 \mathrm{a}, 00 \mathrm{~b}$, and 00c in descending polarized intensity. Several interesting results can be seen in this figure. First of all, as intended, the high resolution VLA observations are resolving structure in the QSO. The combination of the high resolution and simultaneous broad wavelength coverage of S-band allow us to map $\alpha$ across the source. Based on the map of $\alpha$, the QSO appears to be resolved into a flat- $\alpha$ core with a steep- $\alpha$ lobe on either side. As expected, this flat- $\alpha$ core is coincident with the SDSS detection. We can also see that the peaks in polarization are coincident with steeper- $\alpha$. These initial results show that we resolve the RM structure in distant QSOs and will be able to study the intrinsic magnetic field properties of these systems.

In addition to detecting the control QSO in polarization, we detect three new sources of polarized emission. These sources were previously detected in total intensity in the NVSS (Condon et al. 1998), but not in polarized intensity. These serendipitous discoveries will be excluded from the analysis of the intended survey until ancillary data is assembled for identification. However, they may prove useful in estimating the $\mathrm{RM}_{M W}$. The final results of this survey will help to constrain the occurrence of coherent magnetic fields around young, evolving galaxies at $z \sim 0.5$. The methods and techniques used in our survey will pioneer similar types of studies to be done with data from the VLA Sky Survey and the Square Kilometer Array. We are entering a new era of radio polarization observations that will provide opportunities to answer outstanding questions about the origin and evolution of magnetic fields in and around galaxies.

\section{References}

Bernet, M. L., et al. 2008, Nature, 454, 302

Brentjens, M. A. \& de Bruyn, A. G. 2005, A\& $A, 441,1217$

Condon, J. J., et al. 1998, AJ, 115, 1693

Farnes, J. S., et al. 2014, ApJ, 795, 63

O'Sullivan, S. P., et al. 2012, MNRAS, 421, 3300

Rubin, K. H. R., et al. 2010, ApJ, 719, 1503

Schnitzeler, D. H. F. M., Banfield, J. K., \& Lee, K. J. 2015, MNRAS, 450, 3579

Zibetti, S., et al. 2007, ApJ, 658, 161 